\title{
Peritonitis esclerosante primaria y secundaria. Estudio comparativo
}

\author{
Jorge Rojas C. ${ }^{1}$
}

TRABAJO DE INGRESO

'Departamento de Cirugía Hospital Clínico Universidad de Chile. Santiago, Chile.

Recibido el 14 de enero de 2019 y aceptado para publicación el 29 de enero de 2019

Correspondencia a: Dr. Jorge Rojas C. jrojascaro@yahoo.com

\section{Primary and secondary sclerosing peritonitis. Comparative study}

Introduction: There are two types of sclerosing peritonitis (SP): primary or idiopathic and secondary, generally to peritoneal dialysis, and less frequently, to other abdominal or systemic pathologies. Mortality related to this is high. Objective: To compare the clinical feature, diagnostic studies and treatment of patients with Primary and Secondary Sclerosing Peritonitis, to define whether there are any differences and to establish the main clinical and imaging elements allowing for an early diagnosis and improving the therapeutic results. Material and Methods: An analysis of 18 SP cases diagnosed at our hospital between 2001-2014 was carried out. This includes a retrospective series of 15 cases of secondary SP (13 to peritoneal dialysis and 2 to liver cirrhosis). This is compared against a prospective study that includes 3 patients with primary SP. Results: The main differences became evident in the clinical presentation: Primary SP: occurs in an intestinal obstruction and a loss of weight scenario of varying degrees. Secondary SP: abdominal pain and recurrent peritonitis as well as ultrafiltration failure prevail. CT of the abdomen has proven to be useful, in particular in those cases where there is intestinal obstruction. It has made preoperative diagnostic possible. Conclusions: A high degree of suspicion is required for an SP early diagnosis, especially for the primary form. All patients presenting abdominal pain, recurrent vomiting and any degree of weight loss and those with five or more years of peritoneal dialysis presenting abdominal pain and/or recurrent peritonitis and/or ultrafiltration failure should raise a diagnosis suspicion.

Key words: sclerosing peritonitis; intestinal obstruction; cocoon syndrome.

\section{Resumen}

Introducción: Existen dos tipos de peritonitis esclerosante (PE): primaria o idiopática y secundaria, generalmente a diálisis peritoneal (DP), y con menor frecuencia a otras patologías abdominales o sistémicas. Su mortalidad es alta. Objetivo: Comparar las características clínicas, estudios diagnósticos y tratamiento de pacientes con Peritonitis Esclerosante Primaria y Secundaria, definir si existen diferencias y determinar los principales elementos clínicos e imagenológicos que permitan hacer un diagnóstico precoz y mejorar los resultados terapéuticos. Material y Métodos: Se analizan 18 casos de PE diagnosticados en nuestro hospital, entre los años 2001-2014. Incluye una serie retrospectiva de 15 casos de PE secundaria (13 por diálisis peritoneal y 2 por cirrosis hepática). Se compara con un estudio prospectivo que incluye 3 pacientes con PE primaria. Resultados: Las principales diferencias se evidencian en la presentación clínica: PE primaria: se presenta con cuadro de obstrucción intestinal y baja de peso de distinta magnitud. PE secundaria: predominan el dolor abdominal, peritonitis recurrente y la falla de ultrafiltración. La tomografía computada de abdomen es útil, sobre todo cuando hay obstrucción intestinal. Ha hecho posible el diagnóstico preoperatorio. Conclusiones: Se requiere un alto índice de sospecha para el diagnóstico precoz de PE, sobre todo para la forma primaria. Debe sospecharse en todo paciente con dolor abdominal, vómitos recurrentes y baja de peso de cualquier magnitud; y en aquellos en diálisis peritoneal durante 5 años o más, que presenten dolor abdominal y/o peritonitis recurrente y/o falla de ultrafiltración.

Palabras clave: peritonitis esclerosante; obstrucción intestinal; cocoon abdominal. 


\section{Introducción}

Existen dos tipos de peritonitis esclerosante: primaria o idiopática y secundaria ${ }^{1,2}$. La forma secundaria, generalmente a diálisis peritoneal, es la más frecuente y constituye la mayor parte de la experiencia publicada ${ }^{2,3}$. También puede estar relacionada a sarcoidosis, tuberculosis peritoneal, cirrosis, lupus eritematoso sistémico, etc. ${ }^{2,4}$.

La Peritonitis Esclerosante (PE) es una entidad clínica poco frecuente y de alta mortalidad ${ }^{2,4-15}$. Se caracteriza por fibrosis peritoneal visceral que puede ser difusa, nodular o multinodular. En su forma más severa, llamada Peritonitis Esclerosante Encapsulante (PEE) llega a envolver parcial o totalmente las asas intestinales, provocando cuadros de obstrucción o suboclusión intestinal, en forma aguda, subaguda o crónica y generalmente de difícil diagnóstico.

La experiencia publicada se refiere mayormente a la forma secundaria de PE. De la forma primaria se han reportado pocos casos en el mundo. En América hay reportados tres casos en Brasil ${ }^{16,17}$ y 2 en Estados Unidos ${ }^{18}$. En Chile no existen casos publicados.

El objeto del presente trabajo es comparar las características clínicas, estudios diagnósticos y tratamiento de pacientes con PE Primaria y Secundaria, definir si existen diferencias y determinar los principales elementos clínicos e imagenológicos que permitan hacer un diagnóstico precoz y mejorar los resultados terapéuticos.

\section{Material y Métodos}

Se analizan 18 casos de PE diagnosticados en nuestro hospital, entre los años 2001 al 2014. Incluye una serie retrospectiva de 15 casos de PE secundaria (13 a diálisis peritoneal y 2 a cirrosis hepática). Se compara con un estudio prospectivo de 3 pacientes con PE primaria. Los criterios diagnósticos fueron: características macroscópicas del peritoneo al momento de la cirugía y/o cambio o retiro de catéter peritoneal y/o confirmación A-P. Se analizaron las características clínicas, imagenológicas, histológicas, y tratamiento realizado.

Además se revisó la literatura publicada al respecto, hasta la actualidad.

\section{Resultados}

\section{PE Primaria}

\section{Caso 1}

Varón, 18 años, con vómitos y dolor abdominal recurrente de 1 año de evolución, baja de peso (18
$\mathrm{K}$ ), con múltiples consultas y hospitalizaciones y múltiples exámenes radiológicos y endoscópicos entre los que destacan:

Videoendoscopia:

18-02-2008: Esofagitis erosiva grado II de Savary Miller. Gastritis Erosiva antral. Gastritis erosiva fundo-corporal. Cardias amplio incompetente.

10-03-2008: Megaestómago (se aspira contenido bilioso $1100 \mathrm{cc}$, sin grumos a pesar de ayuno). Megaduodeno sin causa obstructiva evidente (se aspira 500 cc más de bilis de duodeno)

Bp 10-03-2008: Lesión ulcerada crónica activa sin elementos de especificidad.

29-05-2008: Esofagitis erosiva distal moderada. Síndrome de retención gástrica-duodenal. Megaduodeno. Obs comprensión extrínseca de cara anterior de duodeno.

\section{TC de Abdomen y pelvis:}

27-05-2008: Significativa dilatación de estómago, duodeno y yeyuno proximal sin evidencias de una causa obstructiva distal a este nivel. No es descartable el fenómeno de pinza mesentérica sobre la $3^{\circ}$ porción duodenal. Se sugiere enteroclisis con TC.

Enteroclisis TC 29-05-2008: Íleo de intestino delgado proximal sin evidencias de obstrucción mecánica. No se identifican hallazgos que sustenten la posibilidad de pinza mesentérica como etiología obstructiva

24-10-2008: Hallazgos de obstrucción intestinal incompleta de larga evolución con megaduodeno secundario a aglomeración de asas de yeyuno e íleon, posiblemente por bridas o en el contexto de una hernia interna (Figuras 1 y 2 ).

Tratamiento: quirúrgico (30-10-2008) buena evolución posterior. Se realizó desbridación con incisión y extirpación del peritoneo fibrosado, liberación de las asas intestinales "encapsuladas" (Figura 3).

Informe A-P: Tejido fibroadiposo congestivo (Figura 4).

La evolución alejada ha sido buena, sin complicaciones, sin recidiva, con recuperación del peso perdido a 7 años de operado.

\section{Caso 2}

Varón, 39 años. Acude al HCUCH con historia de dolor abdominal y vómitos recurrentes, a realizarse examen por el antecedente de: dilatación severa de estómago, duodeno y yeyuno proximal en tomografía computada reciente (07-07-2010). Baja de peso: 29 kilos en 3 meses.

Examen: Enteroclisis TC.

Impresión Tomográfica: 20-07-2010. 


\section{ARTíCULO ORIGINAL}

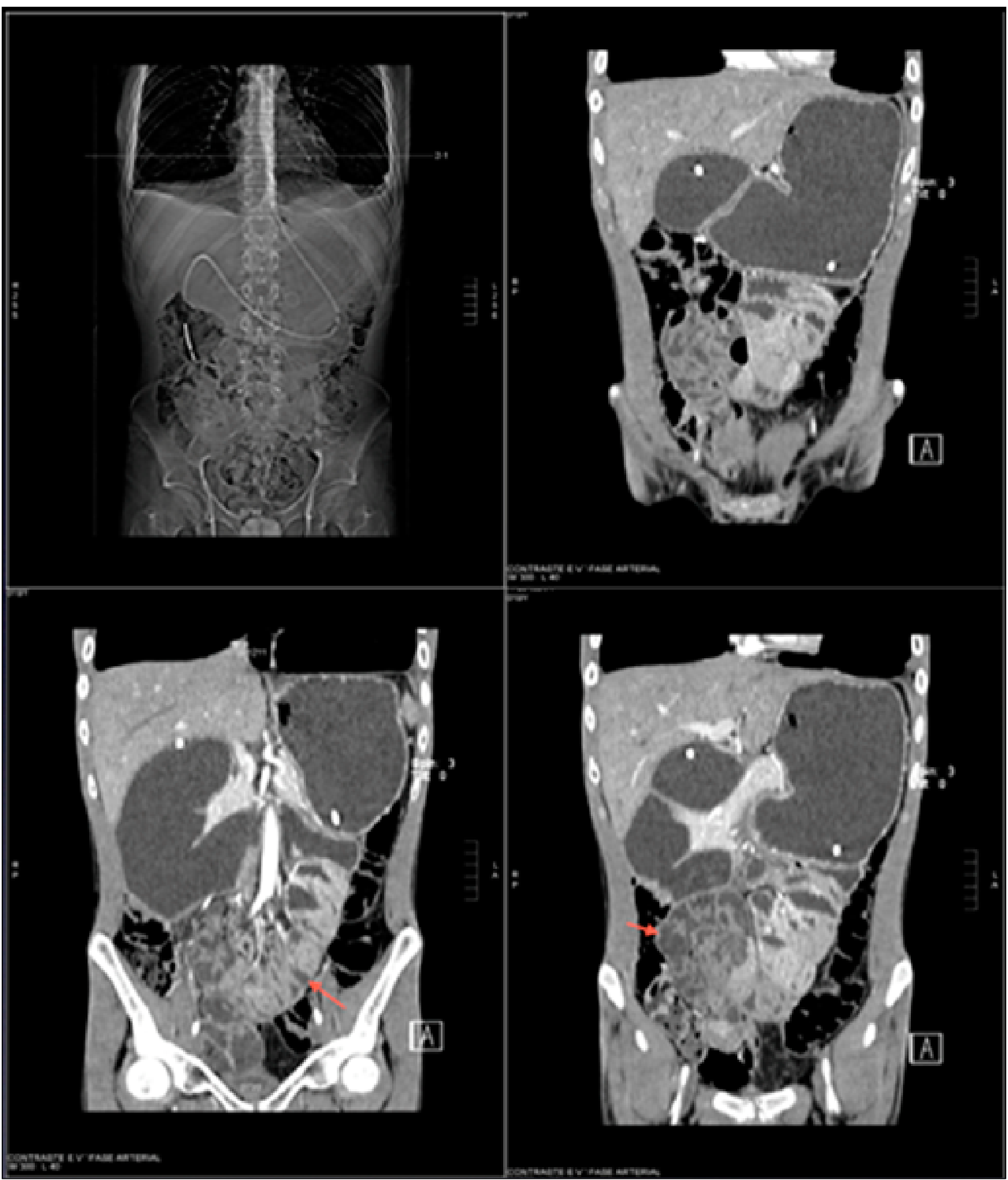

Figura 1. TC Abdomen y Pelvis. Obsérvese la gran dilatación gastroduodenal y la imagen en capullo o cocoon (flechas). 


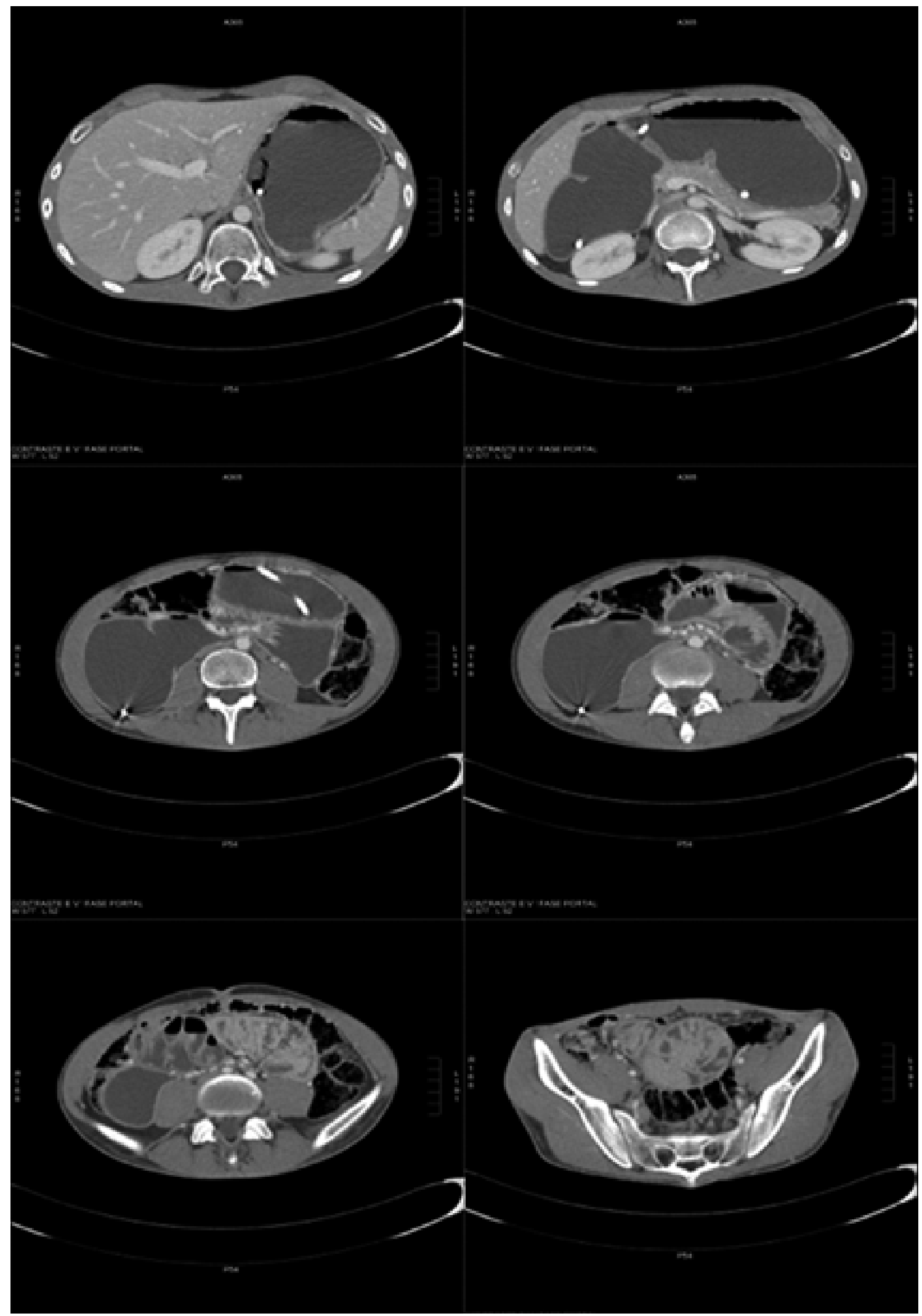

Figura 2. TC de Abdomen y Pelvis, cortes sagitales. 


\section{ARTÍCULO ORIGINAL}

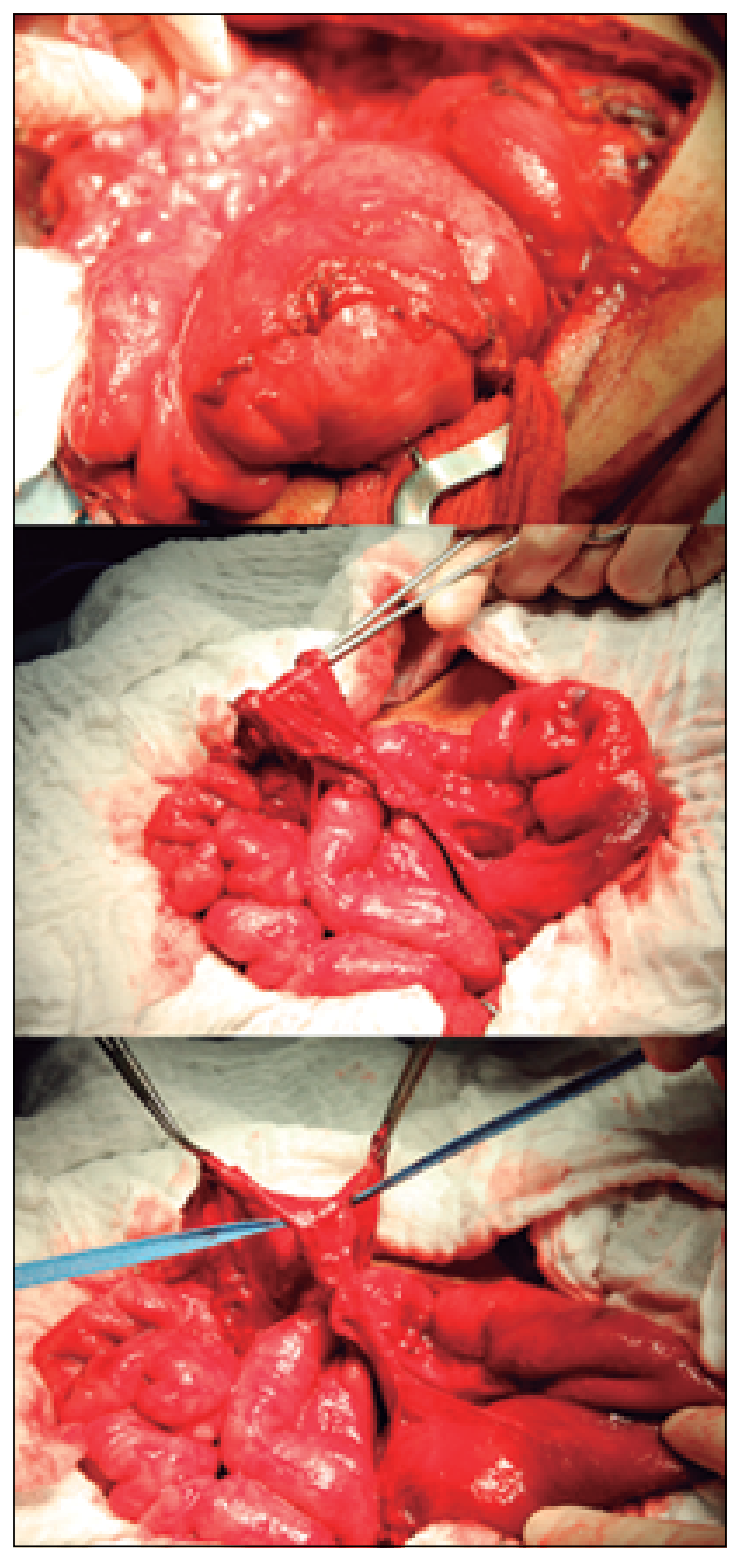

Figura 3. Liberación quirúrgica de las asas intestinales encapsuladas. Tinción $\mathrm{HE}$, hematoxilina y eosina.
Hallazgos compatibles con peritonitis encapsulante que engloba la totalidad de yeyuno e íleon y provoca estenosis intestinal secundaria a compresión de asas, severa dilatación de yeyuno proximal, duodeno y estómago, concordante con proceso de larga evolución.

Videoendoscopia (04-03-2010):

Gastritis Crónica erosiva de $1 / 3$ superior de antro. Cardias amplio.

Tratamiento: quirúrgico (02-08-2010). Buena evolución posoperatoria.

Hallazgo: abdomen sellado por membrana que cubre la totalidad de las asas intestinales encapsulándolas.

\section{Detalle operatorio:}

Enterolisis y extirpación de membrana que sella la totalidad de las asas. Aseo y revisión de hemostasia.

Informe A-P: tejido fibrohialino con revestimiento mesotelial en una de sus caras. No hay signos inflamatorios activos. Sinequias fibrohialinas peritoneales

La evolución alejada ha sido satisfactoria sin complicaciones posoperatorias, ha recuperado su peso y trabaja normalmente a 5 años de operado.

\section{Caso 3:}

Varón, 50 años, con dolor abdominal recurrente de 5 meses de evolución, en ocasiones asociado a ingesta alimentaria, por lo que disminuyó el volumen y frecuencia de la misma. Náuseas y vómitos día por medio, baja de peso (10 kilos) en ese periodo de tiempo.

Antecedentes: Desde hace 8 años meteorismo ante la ingesta de queso, lactosa, frituras, papas, cebolla, uva (ahora come de todo).

Traía:

Videoendoscopia (2):

Mínima metaplasia columnar en esófago inferior (5 mm) (10-01-2011).
Figura 4. Estudio histológico de la membrana peritoneal que muestra infiltrado inflamatorio crónico y proliferación de fibroblastos.

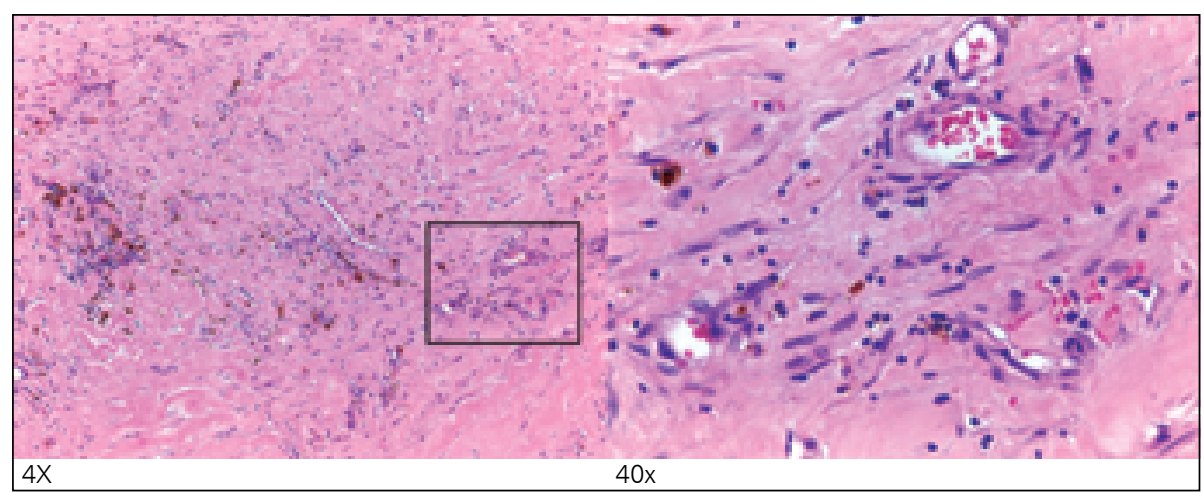



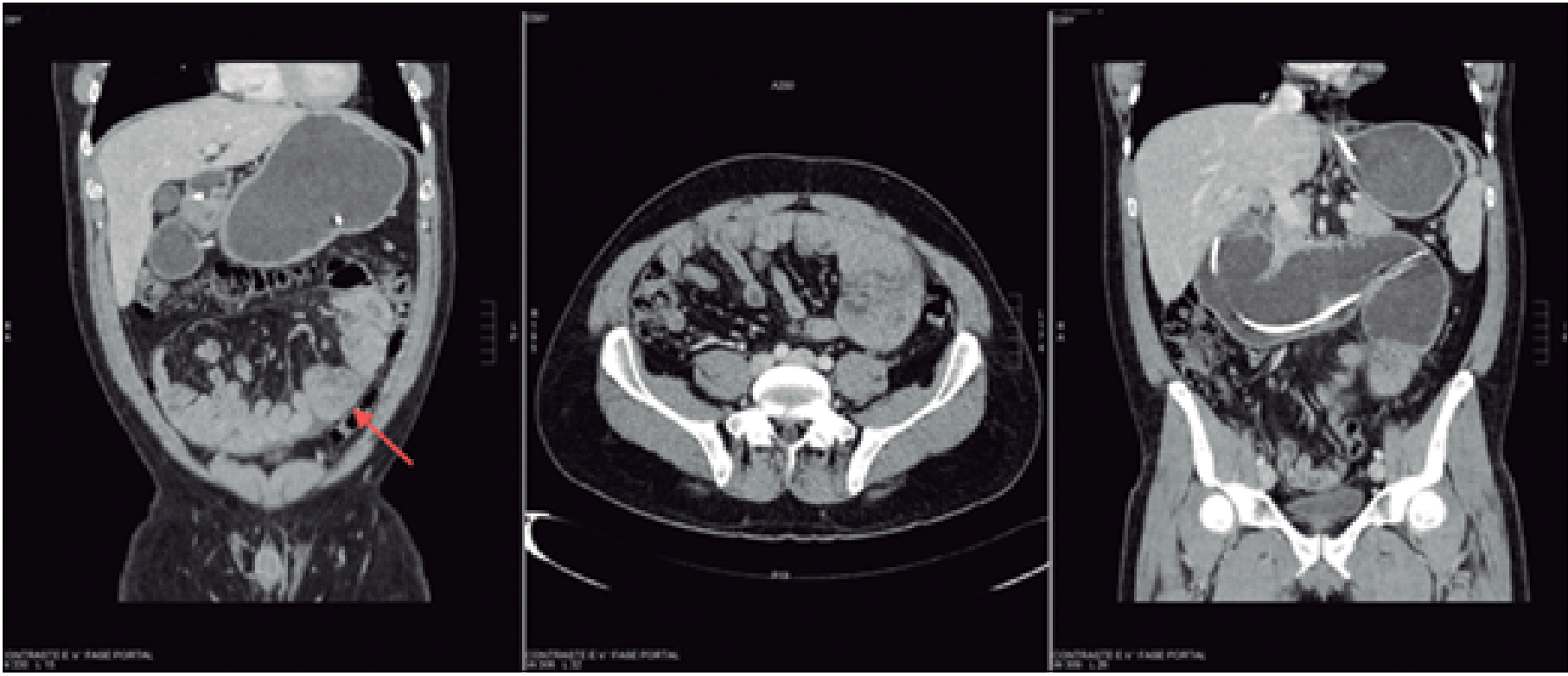

Figura 5. TC de Abdomen y Pelvis, donde es posible apreciar el llamado "capullo" (flecha) y la gran dilatación gastroduodenoyeyunal proximal.

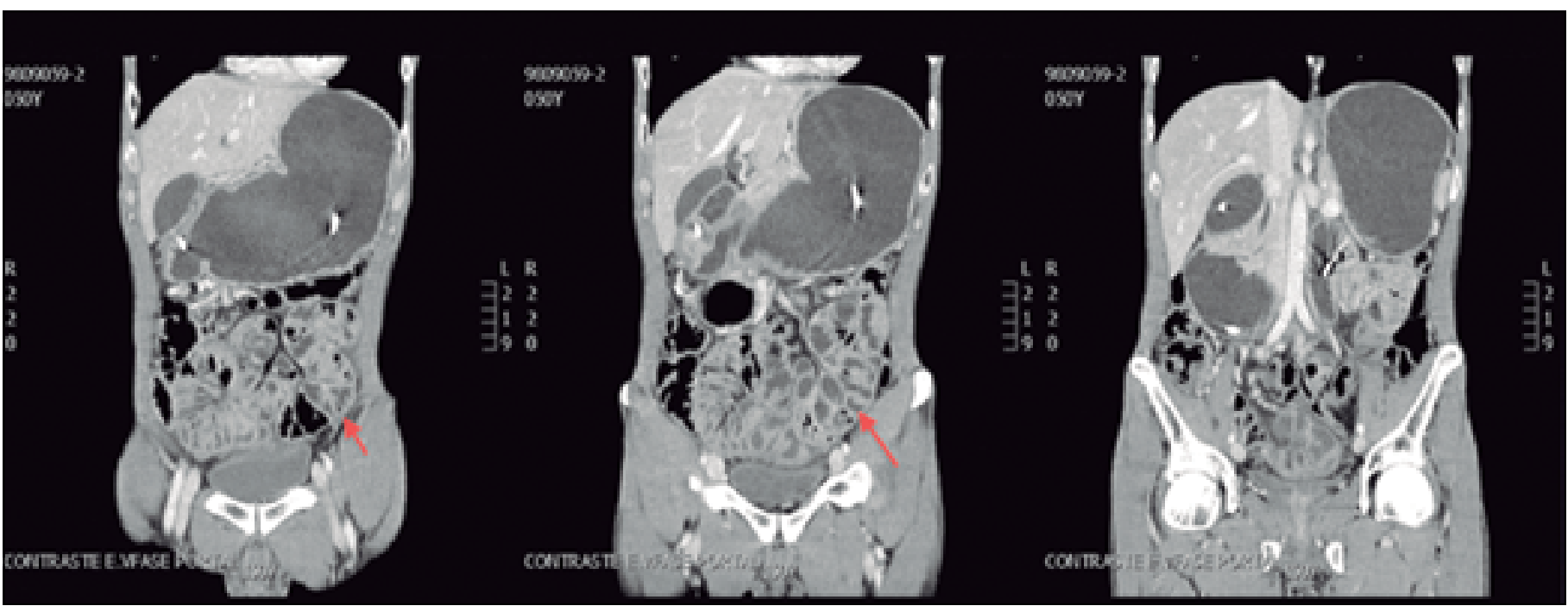

Figura 6. TC de Abdomen y Pelvis mostrando el capullo o cocoon (flecha), además de gran distensión gastroduodenoyeyunal.

Reflujo duodeno-gástrico (27-11-2013).

TC de abdomen y pelvis (16-01-2014):

Hemangioma hepático. "Llama la atención distensión gástrica, de duodeno, yeyuno e íleon sin evidente zona de cambio de calibre". También "es llamativa una relativa aglomeración de asas con una distribución en nido (cocoon), en ambas fosas iliacas con una fina pared que delimita ambas estructuras".

\section{Conclusión}

Hemangioma hepático.

Hallazgos sugerentes de Peritonis Esclerosanta Incapsulata.

Tratamiento: quirúrgico (25-02-2014), con buena evolución posoperatoria. Se realizó liberación de adherencias y extirpación de membrana que envolvía totalmente las asas de intestino delgado (Figura 7).

Informe A-P: Peritonitis Crónica Fibrosante. 


\section{ARTíCULO ORIGINAL}

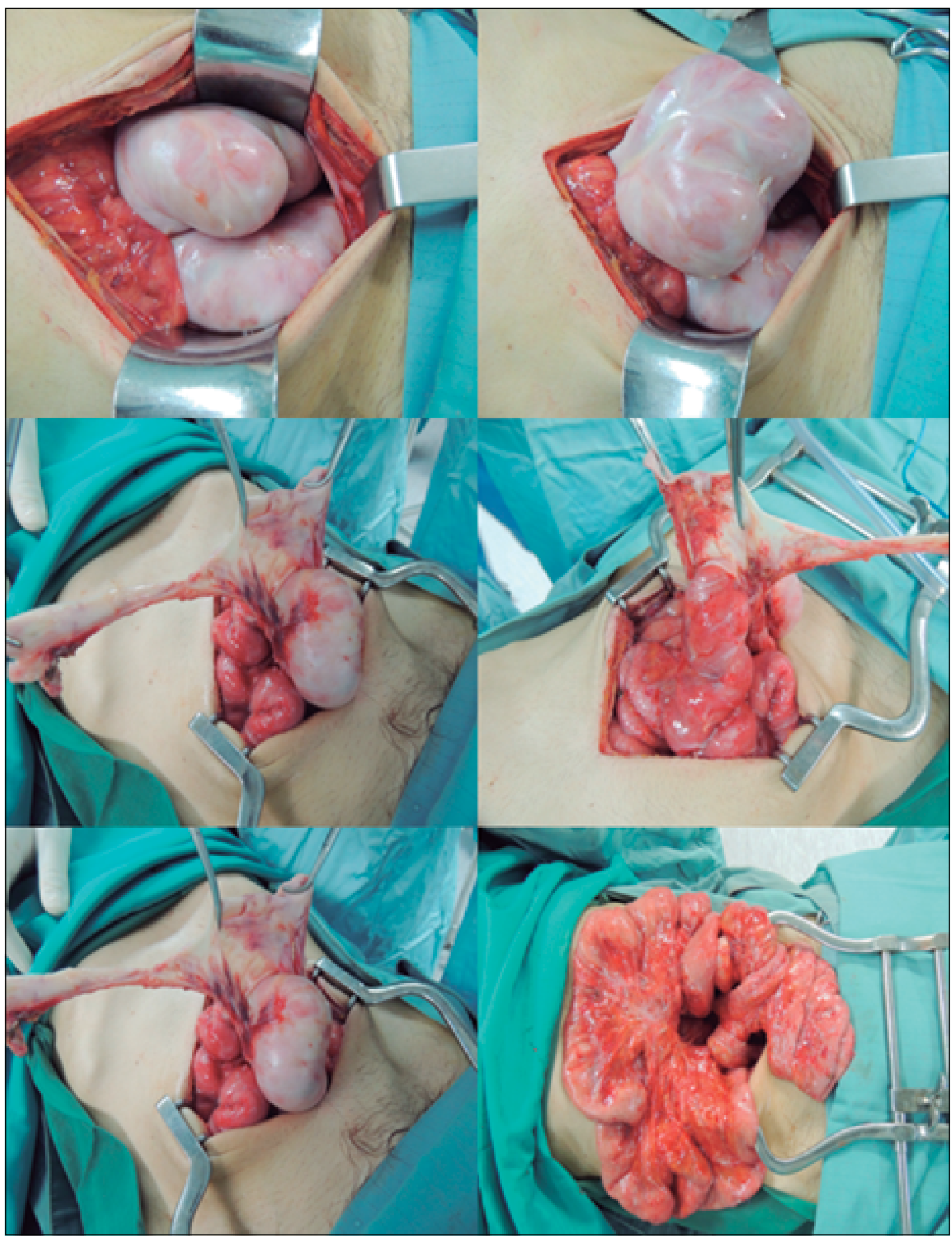

Figura 7. Hallazgos operatorios, con liberación total de asas. 
La evolución alejada fue buena, con remisión total de síntomas y recuperación progresiva de peso a los 17 meses de seguimiento.

\section{PE Secundaria}

15 pacientes tuvieron diagnóstico de PES entre los años 2001 y 2014, con edad promedio de 49,7 años (rango de 28 a 75 años), siendo 9 mujeres $(60 \%)$ y 6 hombres $(40 \%)$.

La enfermedad de base en 13 pacientes $(86,6 \%)$ fue insuficiencia renal crónica y en $2(13,3 \%)$ cirrosis hepática, uno de ellos con antecedente de uso prolongado de propranolol. El tiempo promedio de la diálisis peritoneal fue de 100 meses.

\section{Cuadro Clínico}

Las características clínicas en estos pacientes se detallan en la Tabla 1, en la cual se compara con los 3 pacientes con $\mathrm{PE}$ primaria, observándose claras diferencias. Se consideró peritonitis a repetición el antecedente de 2 o más episodios de peritonitis aguda previa. Este hecho estuvo presente en el 73,3\% de los pacientes con PE secundaria, todos ellos en peritoneodiálisis; sólo en 3 de ellos no existía ningún episodio de peritonitis aguda previa. La obstrucción intestinal en cambio, estuvo presente en todos los casos de PE primaria, y también en los casos de PE secundaria a cirrosis, al igual que síntomas de RGE y baja de peso, mientras que son raros en pacientes con diálisis peritoneal. En este último grupo la peritonitis a repetición, el hemoperitoneo y falla de la ultrafiltración fueron hechos frecuentes. El dolor abdominal recurrente es un síntoma frecuente en los dos grupos.

\section{Estudios Imagenológicos}

Los hallazgos endoscópicos y de imágenes se detallan en la Tabla 2. Estos estudios se realizaron en todos los pacientes con PE primaria y en 14 de los 15 pacientes con PE secundaria. Alteraciones del esófago y estómago sólo se apreciaron en pacientes con PE primaria. El escáner de abdomen mostró signos de obstrucción intestinal, aglomeración de asas y encapsulamiento intestinal en todos los pacientes con PE primaria y PE secundaria a cirrosis y sólo en un porcentaje mucho menor en pacientes en peritoneodiálisis, en este grupo es más probable encontrar engrosamiento peritoneal y calcificaciones (Figura 8).

\section{Histología}

Los hallazgos histológicos se detallan en la Tabla 3,12 de los 15 pacientes con peritonitis secundaria tenían estudio A-P; en los 3 pacientes sin estudio
A-P el diagnóstico se hizo por las características macroscópicas del peritoneo visible durante la cirugía o el cambio/retiro del catéter. En los pacientes con estudio histológico se confirmó PE, con colágeno en el peritoneo. También se describen infiltrado linfoide, y vasos en neoformación. En el grupo de pacientes con PE secundaria se describe también mesotelio atrófico y calcificaciones, ya sea como calcificaciones distróficas focales o como depósitos cálcicos en la pared y lumen de vasos sanguíneos. En los dos grupos se encontró signos de actividad inflamatoria aguda en grado variable.

\section{Tratamiento}

Cuando hubo obstrucción intestinal el tratamiento (Tabla 4) fue quirúrgico. Esto ocurrió en los 3 pacientes $(100 \%)$ con PE primaria y en 4 pacientes

Tabla 1. Características Clínicas. Cuadro Comparativo

\begin{tabular}{|lcc|}
\hline & $\begin{array}{c}\text { Peritonitis Primaria } \\
\mathbf{n}=\mathbf{3}\end{array}$ & $\begin{array}{c}\text { Peritonitis Secundaria } \\
\mathbf{n}=\mathbf{1 5}\end{array}$ \\
\hline Dolor abdominal recurrente & $100 \%$ & $12(80 \%)$ \\
Vómitos & $100 \%$ & $4(26,6 \%)$ \\
\hline Reflujo G-E & $100 \%$ & $2(13,3 \%)$ \\
Baja de peso & $100 \%$ & $3(20 \%)$ \\
Obstrucción Intestinal & $100 \%$ & $4(26,6 \%)$ \\
Peritonitis a repetición & 0 & $11(73,3 \%)$ \\
Hemoperitoneo & 0 & $5(33,3 \%)$ \\
Falla de ultrafiltración & 0 & $8(53,3 \%)$ \\
\hline
\end{tabular}

Tabla 2. Hallazgos Imagenológicos en Peritonitis Esclerosante

\begin{tabular}{|lcc|}
\hline Endoscopía & $\begin{array}{c}\text { Peritonitis } \\
\text { Primaria } \\
\mathbf{n = 3}\end{array}$ & $\begin{array}{c}\text { Peritonitis } \\
\text { Secundaria } \\
\mathbf{n}=\mathbf{1 4}\end{array}$ \\
Esofagitis & $100 \%$ & 0 \\
Gastritis & $100 \%$ & 0 \\
\hline Úlcera & $33 \%$ & 0 \\
Megaestómago & $100 \%$ & 0 \\
TC & & \\
\hline Dilatación estómago duodeno-yeyuno & $100 \%$ & 0 \\
\hline Aglomeración de asas & $100 \%$ & $14,2 \%$ \\
\hline Signos de Obstrucción Intestinal & $100 \%$ & $35,7 \%$ \\
Engrosamiento peritoneal & $100 \%$ & $50 \%$ \\
Encapsulamiento Intestinal & $60 \%$ & $14,2 \%$ \\
\hline Calcificaciones peritoneales & & $42,8 \%$ \\
\hline
\end{tabular}




\section{ARTÍCULO ORIGINAL}

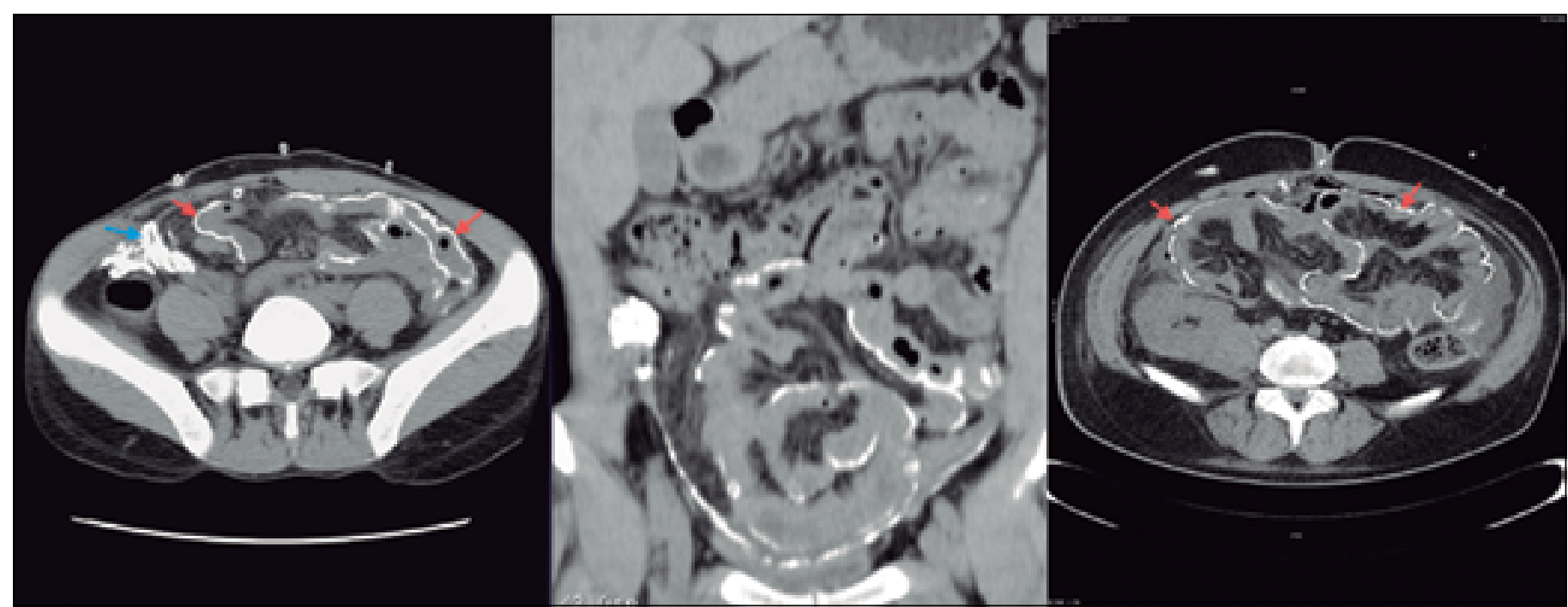

Figura 8. TC de Abdomen y Pelvis mostrando calcificaciones del peritoneo visceral (flechas rojas) y depósitos de calcio amorfos (flecha celeste).

Tabla 3. Estudio Histológico

\begin{tabular}{|lcc|}
\hline & $\begin{array}{c}\text { Peritonitis Primaria } \\
\mathbf{n}=\mathbf{3}\end{array}$ & $\begin{array}{c}\text { Peritonitis Secundaria } \\
\mathbf{n}=\mathbf{1 2}\end{array}$ \\
\hline Fibrosis Peritoneal & $100 \%$ & $100 \%$ \\
Colágeno en Peritoneo & $100 \%$ & $100 \%$ \\
Infiltrado linfoide & $66 \%$ & $58,3 \%$ \\
Vasos en neoformación & $33 \%$ & $41,6 \%$ \\
Calcificaciones & - & $41,6 \%$ \\
Mesotelio atrófico & - & $33,3 \%$ \\
Infiltrado PMN & $33 \%$ & $41,6 \%$ \\
\hline
\end{tabular}

Tabla 4. Tratamiento

\begin{tabular}{|lcc|}
\hline & $\begin{array}{c}\text { Peritonitis Primaria } \\
\mathbf{n = 3}\end{array}$ & $\begin{array}{c}\text { Peritonitis Secundaria } \\
\mathbf{n}=\mathbf{1 5}\end{array}$ \\
Cirugía & $3(100 \%)$ & $4(26,6 \%)$ \\
Tamoxifeno +prednisona & 0 & $10(66,6 \%)$ \\
Retiro de catéter & & $13(86,6 \%)$ \\
\hline
\end{tabular}

Tabla 5. Mortalidad

\begin{tabular}{|lcc|}
\hline & $\begin{array}{c}\text { Peritonitis Primaria } \\
\mathbf{n}=\mathbf{3}\end{array}$ & $\begin{array}{c}\text { Peritonitis Secundaria } \\
\mathbf{n}=\mathbf{1 5}\end{array}$ \\
Mortalidad & 0 & $4(26,6 \%)$ \\
\hline
\end{tabular}

(26,6\%) con PE secundaria, correspondiendo dos de ellos a pacientes en peritoneodiálisis y dos a pacientes con cirrosis hepática (Figura 9). A todos los pacientes que se encontraban en peritoneodiálisis se les retiró el catéter peritoneal y pasaron a hemodiálisis. Además se administró prednisona y tamoxifeno a 10 de ellos, quienes habían mostrado signos de actividad inflamatoria aguda.

De los 4 pacientes fallecidos por PE, dos correspondieron al grupo de peritonitis secundaria a cirrosis hepática, portadores de múltiples patologías, en quienes el tratamiento quirúrgico se realizó cuando ya había necrosis intestinal. Los otros dos pacientes fallecidos pertenecían al grupo de peritonitis secundaria a diálisis peritoneal, uno de ellos desarrolló la enfermedad después haber abandonado la DP, al momento de trasplantarse; la otra paciente falleció por una neumonía+ desnutrición crónica, después de haber dejado la DP y haber pasado a hemodiálisis.

\section{Discusión}

La PE, descrita por primera vez en 1907 por Owtschinnikow, como "peritonitis crónica fibrosa incapsulata"19, es una entidad poco conocida, de baja incidencia y de difícil diagnóstico, descrita principalmente en pacientes sometidos a diálisis peritoneal prolongada y con menor frecuencia asociada a enfermedades en las cuales es esperable cambios inflamatorios peritoneales como la sarcoidosis, 

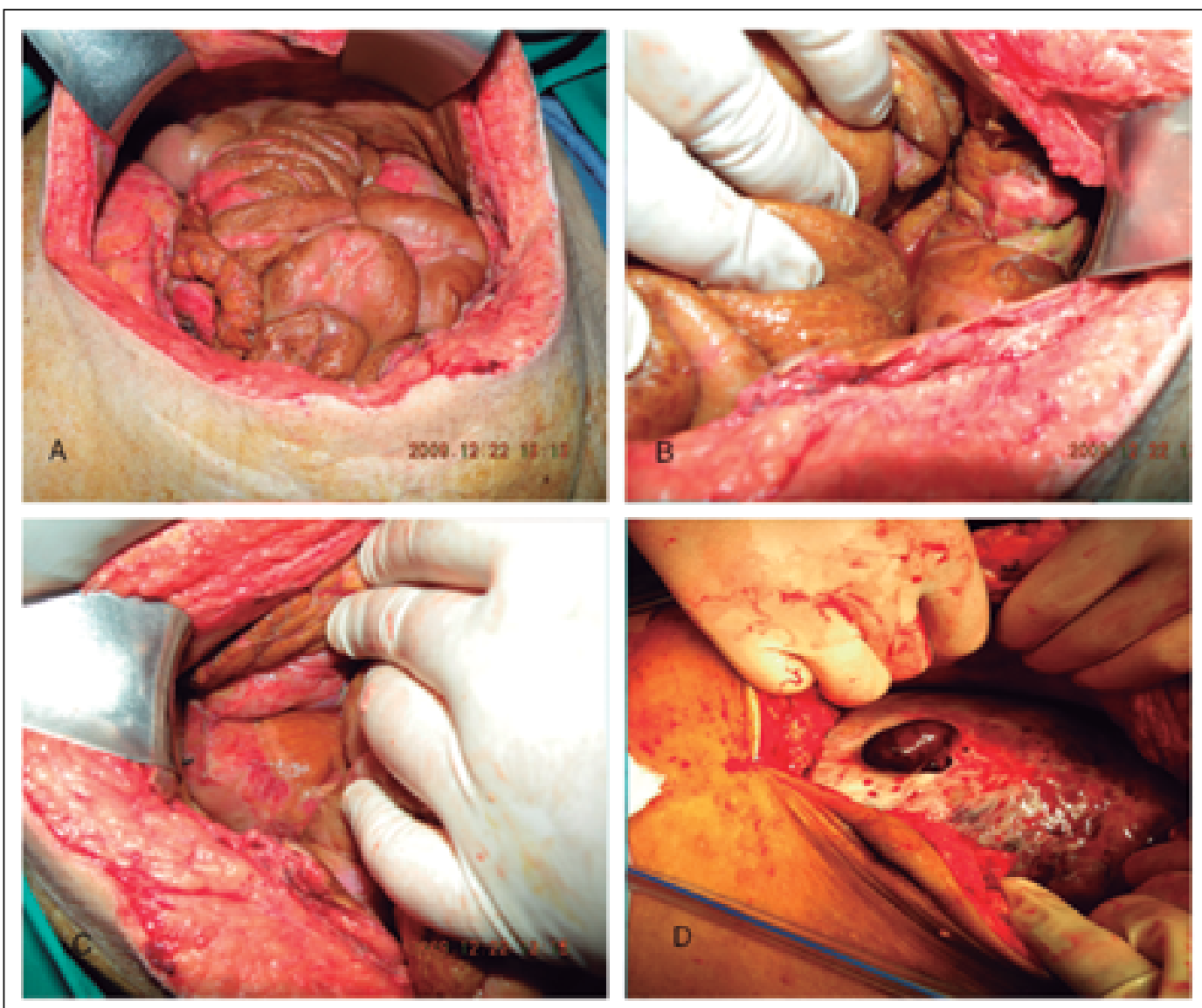

Figura 9. Hallazgos intraoperatorios en peritonitis esclerosante secundaria a diálisis peritoneal $(\mathbf{A}, \mathbf{B}, \mathbf{C})$ y peritonitis esclerosante encapsulante secundaria a cirrosis hepática (D). tuberculosis peritoneal, cirrosis hepática, etc. ${ }^{2,3,9,11}$.

Normalmente el peritoneo consta de una capa de células mesoteliales que descansan sobre una membrana basal debajo del cual hay tejido conjuntivo laxo muy rico en vasos sanguíneos y linfáticos.

En la PE, macroscópicamente, se producen alteraciones anatómicas progresivas de esta membrana, como engrosamiento, adherencias, fibrosis y retracción que puede llegar a comprometer la raíz del mesenterio. Se ha descrito que puede llegar a tener el aspecto de "una suela de zapato desgastado". Histológicamente se produce desmesotelización del peritoneo visceral, con densas capas de tejido fibroconectivo e infiltrado de células mononucleares y polimorfonucleares, con angiogénesis y aumento del número de capilares, fibrina, que puede llegar a la osificación y calcificación.

En los pacientes en peritoneodiálisis, estos cambios inflamatorios estarían desencadenados por la constante exposición del peritoneo a soluciones de glucosa hipertónica ${ }^{20-24}$. Es así que el principal factor de riesgo es el tiempo de permanencia en perito- neodiálisis, con más de 5 años en DP, aumenta en un $14 \%$ el riesgo de desarrollar $\mathrm{PE}^{21}$. La peritonitis aguda a repetición constituye también un factor de riesgo importante.

El tiempo de permanencia en DP fue en promedio 100 meses. Sólo un paciente estuvo menos de 5 años (56 meses), la mayoría más de 8 y hasta 10 años. En cuanto a los cuadros de peritonitis aguda previa, en el 73,3\% de los pacientes con PE secundaria, existía el antecedente de peritonitis a repetición con un promedio de 3 episodios (rango 2-8), correspondiendo todos ellos a pacientes en DP. Además en 5 pacientes $(33,3 \%)$ existía el antecedente de hemoperitoneo.

Los pacientes con PES + Cirrosis hepática presentaron ascitis de larga evolución, uno de ellos cursó con peritonitis bacteriana primaria y en el otro había el antecedente de uso prolongado de propranolol, lo cual también está descrito como uno de los factores relacionados con el desarrollo de peritonitis esclerosante ${ }^{2}$.

Por otro lado la forma primaria o idiopática, menos frecuente aún, es de origen desconocido. 
Fue denominada por Foo como abdominal cocoon síndrome en $1978^{19}$ y se ha descrito en mujeres jóvenes de climas tropicales y subtropicales. Se ha postulado la menstruación retrógrada a través de las trompas de Falopio con una infección viral superpuesta, como causa de $\mathrm{PE}^{19}$. Sin embargo, la PE también se desarrolla en hombres y niños, lo que hace poco probable esta teoría. También se ha sugerido una etiología infecciosa probablemente viral en estos casos. En los 3 pacientes con PE primaria del presente estudio, no existía ningún antecedente infeccioso que pudiera estar relacionado.

Respecto al cuadro clínico, se ha descrito como poco claro, inespecífico. Adicionalmente su baja prevalencia y el escaso conocimiento de esta entidad dificultan el diagnóstico precoz.

En el presente estudio se evidencia claras diferencias en la presentación clínica en las dos formas de PE: En la forma primaria se presenta como obstrucción intestinal con dolor abdominal y vómitos recurrentes y baja de peso variable. El RGE también es importante. En la forma secundaria, predominan el dolor abdominal recurrente, la falla de ultrafiltración (UF) y el antecedente de peritonitis a repetición, la obstrucción intestinal se presentó sólo en el 26,6\% de los casos, que corresponden a 4 pacientes, dos de ellos en DP y dos con cirrosis hepática. Esto se explicaría porque la diálisis peritoneal permite evidenciar cambios en el líquido peritoneal y fallas en la UF, con fácil acceso a una biopsia peritoneal que permite hacer diagnóstico de PE antes de que provoque un cuadro de obstrucción intestinal. De lo contrario probablemente las manifestaciones serían similares a la forma primaria.

En cuanto a los exámenes auxiliares es llamativa la presencia de un Megaestómago en la endoscopía alta en todos los pacientes con PES primaria. En la TC se evidencian signos de obstrucción intestinal en el $100 \%$ de los pacientes, con dilatación de estómago, duodeno y yeyuno. Aun cuando se dice que estos signos tomográficos son tardíos, se puede decir que la TC ha hecho posible el diagnóstico preoperatorio. En los pacientes con PE secundaria la utilidad diagnóstica cuando no había obstrucción intestinal fue menor.

En caso de existir obstrucción intestinal, el tratamiento es quirúrgico y consiste en la liberación del intestino con adherenciolisis, pudiendo ser necesaria la resección intestinal de asas comprometidas.

En pacientes con DP es mandatorio la suspensión de ésta con retiro del catéter. Además se recomienda el uso de corticoides si hay parámetros de actividad inflamatoria, como aumento de la PCR, aunque según un estudio japonés ${ }^{22}$, siempre habría algún grado de actividad inflamatoria, por lo que siempre estaría recomendado su uso, solo o en asociación con tamoxifeno, que tendría menos efectos colaterales.

\section{Conclusiones}

Se requiere un alto índice de sospecha para el diagnóstico precoz de $\mathrm{PE}$, sobre todo para la forma primaria.

Debe sospecharse en todo paciente con dolor abdominal y vómitos recurrentes con baja de peso de cualquier magnitud y en aquellos en diálisis peritoneal durante 5 años o más, que presenten dolor abdominal $\mathrm{y} / \mathrm{o}$ peritonitis recurrente $\mathrm{y} / \mathrm{o}$ falla de ultrafiltración.

Debe considerarse el uso de corticoides solo o en asociación con tamoxifeno como parte del tratamiento.

\section{Responsabilidades éticas}

Protección de personas y animales. Los autores declaran que para esta investigación no se han realizado experimentos en seres humanos ni en animales.

Confidencialidad de los datos. Los autores declaran que en este artículo no aparecen datos de pacientes.

Conflictos de interés: no hay.

\section{Bibliografía}

1. Mohanty D, Jain BK, Agrawal J, Gupta A, Agrawal V. Abdominal cocoon: clinical presentation, diagnosis, and management. J Gastrointest Surg. 2009;13:1160-2.

2. Akbulut S. Accurate definition and management of idiopathic sclerosing encapsulating peritonitis. World J Gastroenterol. 2015;21:675-87.

3. De Souza E, Del Peso-Gilsanz G, Bajo-Rubio MA, Ossorio-González M, Selgas-Gutiérrez R. Peritonitis esclerosante encapsulante asociada a la diálisis peritoneal. Una revisión y una iniciativa unitaria europea para abordar el cuidado de una enfermedad rara. Nefrología 2012;32:707-14.

4. Kaushik R, Punia RPS, Mohan H, Attri AK. Tuberculosis abdominal cocoon- a report of 6 cases and review of the literature. W J Emerg Surg. 2006;1:1820. 
5. Xu P, Chen LH, Li YM. Idiopathic sclerosing encapsulating peritonitis (or abdominal cocoon): a report of 5 cases. World J Gastroenterol. 2007;13:3649-51.

6. Bas G, Eryilmaz R, Okan I, Somay A, Sahin M. Idiopathic abdominal cocoon: report of a case. Acta Chir Belg. 2008;108:266-8.

7. Singh O, Gupta S, Shukla S, Mathur R. Idiopathic sclerosing encapsulating peritonitis; rare cause of intestinal obstruction. Int J Surg. 2008;20:169-71.

8. Zheng YB, Zhang PF, Ma S, Tong SL. Abdominal cocoon complicated with early postoperative small bowel obstruction. Ann Saudi Med. 2008;28:294-6.

9. Choudhury T, Kamal M. Abdominal Cocoon - A Case Report with Short Review of Literature. BSMMU J. 2009;2:81-4.

10. Ibrahim NA, Oludara MA. Abdominal cocoon in an adolescent male patient. Trop Doct. 2009;39:254-6.

11. Kumar A, Ramakrishnan TS, Sahu $\mathrm{S}$, Mishra KB. Idiopathic sclerosing encapsulating peritonitis-is a preoperative diagnosis possible? Report of three cases. Surg Today 2009;39:610-4.

12. Awe JA. Abdominal cocoon syndrome (idiopathic sclerosing encapsulating peritonitis): how easy is its diagnosis preoperatively? A case report. Case Rep Surg. 2013;2013:604061.
13. Pamo Reyna O, Loza Oropeza D, Sáenz Rodriguez M, Chian García C, Verona Rubio R, Freundt Serpa N, et al. Peritonitis Esclerosante: Presentación de tres casos. Rev. Gastroenterol. Perú 2010;30:65-72.

14. Rasihashemi SZ, Ramouz A, Ebrahimi F. An unusual small bowel obstruction (abdominal cocoon): a case report. Arq Bras Cir Dig. 2014;27:82-3.

15. Tannoury JN, Abboud BN. Idiopathic sclerosing encapsulating peritonitis: abdominal cocoon. World J Gastroenterol. 2012;18:1999-2004

16. Araujo Filho JAB, Martines JAS, Martines BMR, Silva AF, Lovisolo $\mathrm{SM}$, Castro CC. Idiopathic sclerosing encapsulating peritonitis: an uncommon cause of intestinal obstruction. Autopsy Case Rep. 2012;2:51-6.

17. Da Luz MM, Barral SM, Barral CM, Bechara Cde S, Lacerda-Filho A. Idiopathic encapsulating peritonitis: report of two cases. Surg Today 2011;41:1644-8

18. Cai J, Wang Y, Xuan Z, Hering J, Helton S, Espat NJ. The abdominal cocoon: a rare cause of intestinal obstruction in two patients. Am Surg. 2007;73:1133-5.

19. Foo KT, Ng KC, Rauff A, Foong WC, Sinniah R. Unusual small intestinal obstruction in girls: The abdominal cocoon. Br J Surg. 1978;65:427-30.
20. Yamamoto R, Nakayama M, Hasegawa T, Miwako N, Yamamoto H, Yokoyami $\mathrm{K}$, et al. High-transport membrane is a risk factor for encapsulating peritoneal sclerosis developing after long-term continuous ambulatory peritoneal dialysis treatment. Adv Perit Dial. 2002;18:131-4.

21. Gayomali C, Hussein U, Protopapas $Z$, Finkelstein FO. Incidence of encapsulating peritoneal sclerosis: a single- center experience with long-term peritoneal dialysis in the United States. Peritoneal Dial Int. 2011;31:279-86.

22. Kawanishi H, Kawaguchi Y, Fukui H, Hara S, Imada A, Kubo H, et al. Encapsulating peritoneal sclerosis in Japan: A prospective, controlled, multicenter study American Journal of Kidney Diseases 2004;44:729-37.

23. Nomoto Y, Kawaguchi Y, Kubo H, Hirano H, Sakai S, Kurokawa K: Sclerosing encapsulating peritonitis in patients undergoing continuous ambulatory peritoneal dialysis: a report of the Japanese Sclerosing Encapsulating Peritonitis Study Group. Am J Kidney Dis. 1996;28:420-7.

24. Soria M, Arrieta J, Ugarte I, Moina I, Guerra M, Escanero J. Alteraciones del pH intracelular en fibroblastos peritoneales inducida por factores implicados en la esclerosis peritoneal. Nefrología 2004;24:158-66.

\section{Comentario al Trabajo de Ingreso}

Quiero agradecer a la Sociedad de Cirujanos por permitirme el honor de comentar el trabajo del Dr. Jorge Rojas con el cual opta para ser aceptado como Miembro Titular y a quién conozco ya por varios años.

El Dr. Rojas nos trae a discusión el tema de la Peritonitis Esclerosante (PES), que aunque de baja frecuencia es muy interesante por el desafío diagnóstico y la complejidad terapéutica que implica.

Como el Dr. Rojas muy bien señala esta entidad constituye una forma anátomo-patológica y clínica de peritonitis crónica muy lejana del componente bacteriano que poseen las peritonitis agudas que enfrentamos más frecuentemente en la práctica diaria. Se ha acuñado esta denominación porque se asocian las características de esclerosante y encapsulante debido a la progresiva formación de tejido fibro-colágeno denso que sella la cavidad perineal involucrando el intestino y generando obstrucción y/o alteración de la motilidad. Lo de peritonitis se refiere al frecuente hallazgo de tejido inflamatorio mononuclear en medio del tejido fibroso peritoneal.

Nos presenta 3 casos de PES primaria también conocida como Peritonitis encapsulata o Cocoon abdominal Syndrome, todos hombres, tendencia que se repite en la literatura y que pone en duda algunas teorías iniciales en que se consideraba más frecuente en mujeres jóvenes especialmente de áreas tropicales.

Los elementos sintomáticos centrales de esta patología son: dolor abdominal crónico, vómitos 
iterativos y baja de peso. Como el Dr. Rojas ha mencionado puede también encontrarse dilatación duodenal y gástrica expresión de obstrucción crónica y que llevan frecuentemente a descartar síndromes de ectasia gástrica por obstrucción duodenal como así también alteraciones funcionales como la miopatía visceral difusa.

Descartado lo anterior, los elementos clínicos centrales del cuadro nos deben hacer pensar en una obstrucción intestinal alta de curso insidioso o crónico. Tratándose de pacientes que no tienen cirugías anteriores ni hernias de la pared abdominal, causas que dan cuenta de 70 a $85 \%$ de las obstrucción intestinal altas, debemos entonces pensar y descartar etiologías menos frecuentes de obstrucción como son el íleo biliar, los tumores de intestino delgado, hernias internas, bridas congénitas etc., todas causas de baja frecuencia y dentro de las cuales está también la PES que la serie del Dr. Rojas pone en la palestra y que sin duda incidentalmente alguna vez a varios nos ha tocado enfrentar .

La PES secundarias tienen diversos orígenes existiendo factores sistémicos que generan fibrosis peritoneal como uso crónico de fármacos ( betabloqueadores, metotrexato y otros.) o bien exposición a formas de irritación peritoneal crónica ya sea de origen química o bacteriana las cuales generan una suerte de respuesta inflamatoria crónica de la serosa peritoneal con generación de fibrosis que puede conducir a bloqueo de la cavidad abdominal o bien a la complicación mayor que es la obstrucción intestinal. La serie del Dr. Rojas muestra que la dificultad progresiva para el uso de catéteres peritoneales constituye el primer elemento que debe hacer sospechar la progresiva generación de una PES.

Sin duda que el estudio con TC y especialmente asociada a Enteroclisis tiene plena indicación. $\mathrm{Su}$ alto rendimiento en demostrar el fenómeno obstructivo ayuda a fundamentar el diagnóstico y aporta frecuentemente información sobre la causa etiológica de la obstrucción. En la PES este estudio posee un gran valor como lo muestran los casos presentados en esta serie y refrendado por la literatura publicada sobre el tema. A pesar de lo anterior cerca de la mitad de los casos de PES siguen llegando a cirugía sin un diagnóstico claro de la verdadera causa obstructiva.

Cuando existe obstrucción intestinal secundaria a una PES sin duda que se constituye un gran desafío técnico. La enterolisis es sin duda difícil y laboriosa por la pérdida de los planos anatómicos generado por la gran fibrosis de dificultan en extremo la disección momentos en se debe ser muy cuidadoso para evitar el trauma quirúrgico sobre las paredes intestinales que hagan necesarias resecciones intestinales mayores o que puedan conducir al gran fantasma que son las fístulas posoperatorias. La serie reportada por el Dr. Rojas tiene muy buenas cifras en este sentido, con escasa morbilidad quirúrgica y buenos resultados a largo plazo especialmente en los casos de PES primarias.

La recurrencia de la obstrucción intestinal en los casos de PES es otra situación descrita y que sin duda incrementa la dificultad del manejo y ensombrece el pronóstico. Como ya se comentara se han empleado diversos fármacos como los corticoides, el tamoxifeno, colchicina etc con las idea de reducir la regeneración de fibrosis peritoneal, pero sus resultados parecen ser bastante empíricos.

Estimado Sr. Presidente, creo que el Dr. Rojas cumple a cabalidad con los requisitos técnicos, científicos y humanos para ser aceptado como Miembro Titular de la Sociedad de Cirujanos de Chile y estoy seguro que esto constituirá un aliciente para que incremente su ya importante aporte a nuestra Sociedad.

Dr. Carlos Benavides 